Utah State University

DigitalCommons@USU

8-2018

\title{
Ultraviolet Diffraction Assisted Image Correlation (UV-DAIC) for Single-Camera 3D Strain Measurement at Extreme Temperatures
}

\author{
Ethan K. Nickerson \\ Utah State University
}

Follow this and additional works at: https://digitalcommons.usu.edu/etd

Part of the Mechanical Engineering Commons

\section{Recommended Citation}

Nickerson, Ethan K., "Ultraviolet Diffraction Assisted Image Correlation (UV-DAIC) for Single-Camera 3D Strain Measurement at Extreme Temperatures" (2018). All Graduate Theses and Dissertations. 7093.

https://digitalcommons.usu.edu/etd/7093

This Thesis is brought to you for free and open access by the Graduate Studies at DigitalCommons@USU. It has been accepted for inclusion in All Graduate Theses and Dissertations by an authorized administrator of DigitalCommons@USU. For more information, please contact digitalcommons@usu.edu.

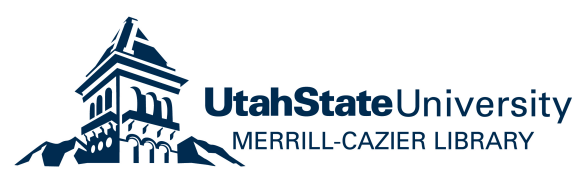


ULTRAVIOLET DIFFRACTION ASSISTED IMAGE CORRELATION (UV-DAIC) FOR SINGLE-

CAMERA 3D STRAIN MEASUREMENT AT EXTREME TEMPERATURES

By

Ethan K. Nickerson

A thesis submitted in partial fulfillment of the requirements for the degree

of

MASTER OF SCIENCE

in

Mechanical Engineering

Approved:

Ryan B. Berke, Ph.D.

Nicholas A. Roberts, Ph.D.

Major Professor

Committee Member

Tadd T. Truscott, Ph.D.

Mark R. McLellan, Ph.D.

Committee Member

Vice President for Research and

Dean of the School of Graduate Studies

UTAH STATE UNIVERSITY

Logan, Utah 
Copyright (C) Ethan K. Nickerson 2018

All Rights Reserved 


\begin{abstract}
Ultraviolet Diffraction Assisted Image Correlation (UV-DAIC) for Single
\end{abstract}

Camera 3D Strain Measurement at Extreme Temperature

by

Ethan K. Nickerson, Master of Science

Utah State University, 2018

Major Professor: Ryan B. Berke, Ph.D.

Department: Mechanical and Aerospace Engineering

Diffraction Assisted Image Correlation (DAIC) measures three-dimensional (3D) full-field deformations using a single camera. This is accomplished by placing transmission diffraction gratings between the specimen and camera to produce multiple views of the specimen at different angles. The angle at which the image appears depends on the wavelength of light used for illumination, the pitch of the gratings being used, and the diffraction order of the image. In this work, DAIC was modified for use at a higher range of temperatures by using ultraviolet (UV) light for illumination and filtering out the visible spectrum light emitted by high temperature samples. These images were then used for 3D displacement and strain measurements using stereo digital image correlation (3DDIC). This method was first tested at room temperature by comparing results to known deformation applied as rigid body motion. It was then demonstrated to be effective in making displacement and strain measurements up to a temperature of $900^{\circ} \mathrm{C}$.

(40 pages) 


\section{PUBLIC ABSTRACT}

Ultraviolet Diffraction Assisted Image Correlation (UV-DIC) for Single

\section{Camera 3D Strain Measurement at Extreme Temperature}

Ethan K. Nickerson

Digital Image Correlation (DIC) is a technique which uses images taken before and after deformation to determine displacement and strain data over the surface of the sample. In order to obtain this data for both in-plane as well as out-of-plane direction, multiple views of the sample are required. Typically, this is accomplished using multiple cameras, but it is possible to use diffraction gratings to bend the light coming from the specimen in order to allow a single camera to capture multiple views. This technique is referred to as Diffraction Assisted Image Correlation (DAIC) and has been previously demonstrated at room temperature. This work expands this method for use at high temperatures by incorporating the use of ultraviolet (UV) lights for illumination and filtering out the light in the visible spectrum. This increases the temperature at which useful images can be captured by reducing the glow that specimens produce at elevated temperatures. When not filtered out, this glow saturates the camera sensor making DIC impossible. This new technique is referred to as Ultraviolet Diffraction Assisted Image Correlation (UV-DAIC). 


\section{ACKNOWLEDGMENTS}

I would like to thank Dr. Berke for his constant help and encouragement from the moment he hired me as an undergraduate researcher to the completion of this thesis. I would especially like to thank him for taking the time to teach me so much along the way. I would like to acknowledge the other members of my committee for their contributions and willingness to help me. I would like to thank the other students in the Mechanics at Extreme Temperature lab for allowing me to bounce ideas off them and willingness to talk through problems I ran into during the course of this research. I would also like to thank my family for their support and encouragement. Finally, I would like to thank the US Nuclear Regulatory Commission for supporting this work under award number NRCHQ-84-15-G0033.

Ethan Nickerson 
CONTENTS

Page

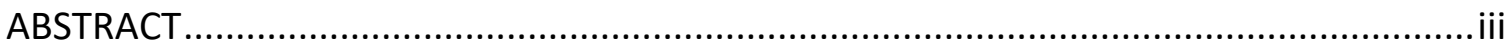

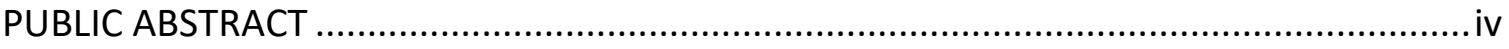

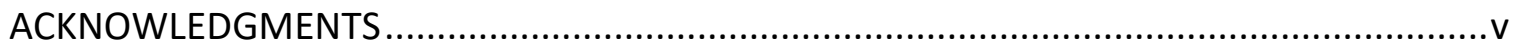

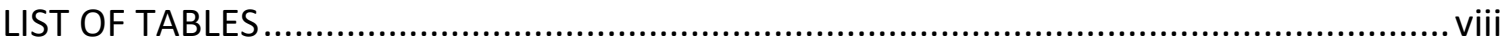

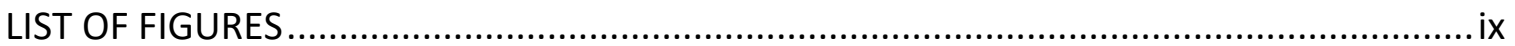

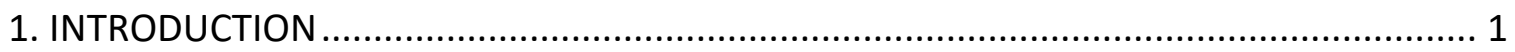

1.1 Overview of Digital Image Correlation ................................................................ 1

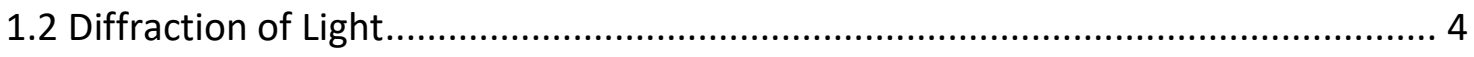

1.3 Diffraction Assisted Image Correlation ............................................................ 6

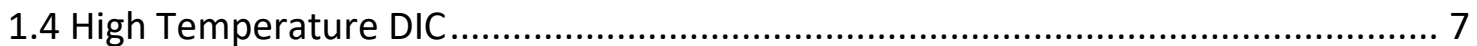

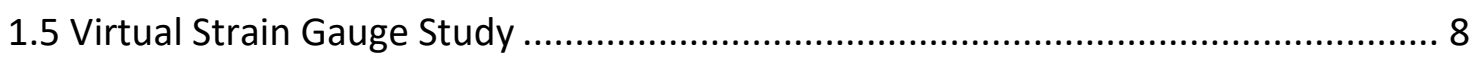

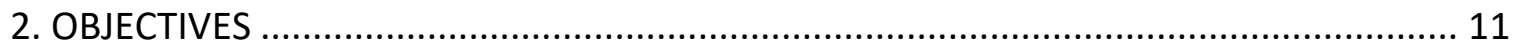

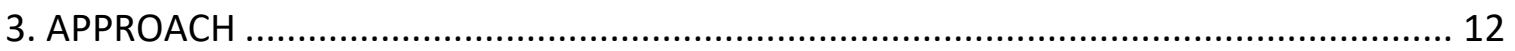

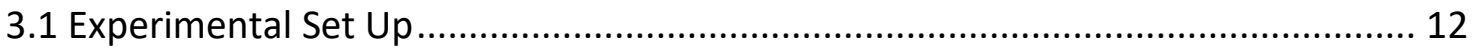

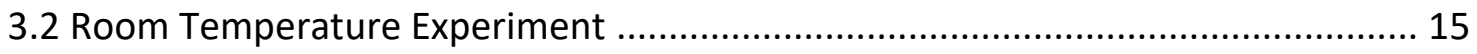

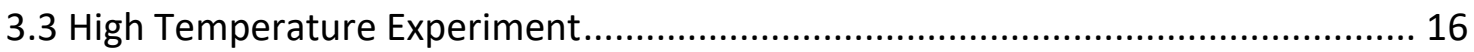

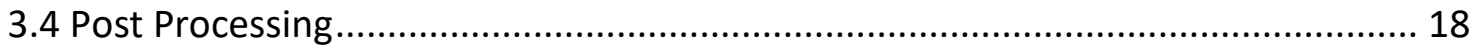

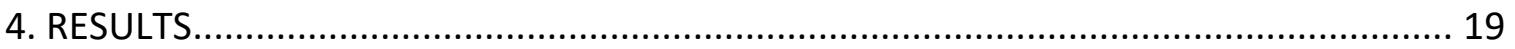

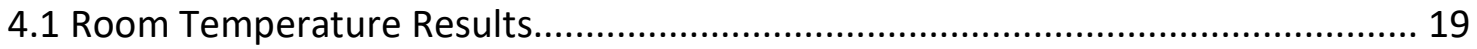

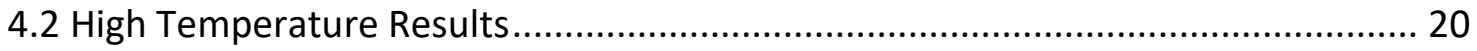

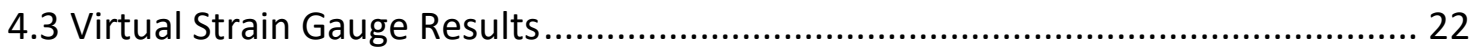

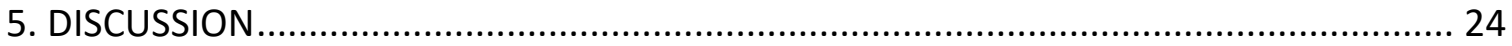

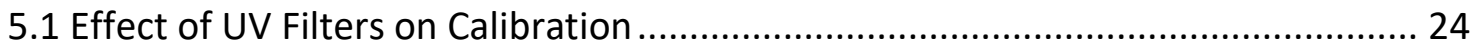

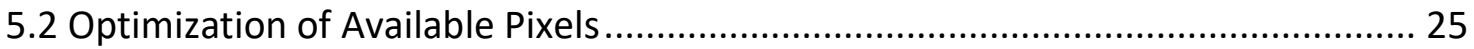




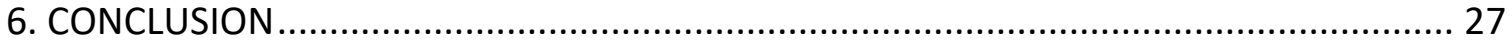

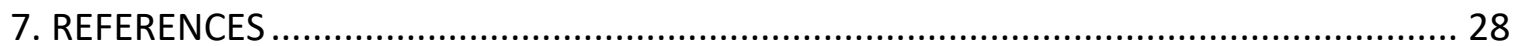




\title{
LIST OF TABLES
}

\author{
Table
}

Page

1 Parameters used to perform stereo DIC 18 


\section{LIST OF FIGURES}

Figure

Page

1 Example patterns for DIC created by (a) stamping, (b) splattering, (c) airbrush, and (d) sponges

2 Views of a stereo calibration grid captured by two different cameras

3 A representation of a wave encountering a barrier with a slit close in size to the wavelength of the wave(left) a barrier with 2 slits resulting in interference (right)

$4 \quad$ Typical groove geometry for transmission grating (a) and blazed grating (b) $m$ denotes the diffraction order. $\theta_{B}$ is the blaze angle

5 Optical arrangement for use of DAIC to observe two stereo perspectives with a single camera

6 An array of subsets used to make a strain measurement. The center of each

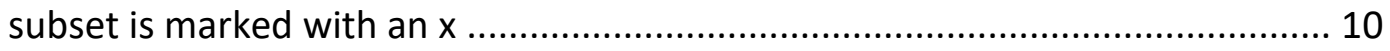

$7 \quad$ Front view of experimental set up with two diffraction gratings outlined by

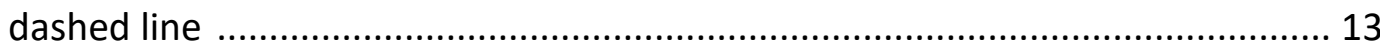

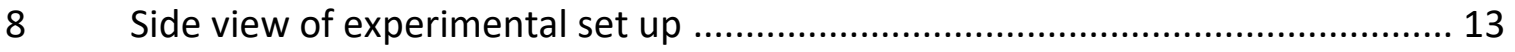

9 Transmission curves for UV optics and camera used in the experiment 15

10 An example of the two first order images captured using a pair of blazed gratings and a single camera the zeroth order image is barely visible in the center of the image. 
11 Temperature Profile in ${ }^{\circ} \mathrm{C}$ captured using IR camera, the outline of the specimen is shown by the dashed line

12 Plot of measured vs applied displacements as well as the percent error between these displacements in the in-plane direction (X) (a) and (c) as well as the out-of-plane direction ( $Z$ ) (b) and (d). The dashed red line represents perfect agreement between the applied and measured displacement. Each measurement is the mean value for the entire region of interest, with uncertainty bands showing two standard deviations

13 The normal strain field in both the $x$ and $y$ directions at the temperature distribution in Figure 11

14 The out-of-plane displacement of the specimen at the temperature shown in Figure 11 (a); and a plot of the out of plane displacement of the specimen along the dashed white line as temperature increased (b). The temperatures given in the legend are the peak temperatures recorded in the specimen ........ 22

15 A plot of the strain in the $y$ direction with the position of the line scan shown (a) Strain calculated along this line using different DIC settings (b). The legend shows the settings in the format SS_ST_SW_VSG. The relative gauge size for each result is shown on the left side

16 Images of calibration grid with various filters. Single broad pass (a), Single narrow filter (b), both in series (c) 


\section{CHAPTER 1}

\section{INTRODUCTION}

\subsection{Overview of Digital Image Correlation}

In order to characterize materials which can withstand extreme temperature environments, such as those used in spacecraft reentry[1], [2], aircraft in hypersonic flight[3], [4], and very high temperature nuclear reactors[5], [6], the ability to measure 3D heterogeneous deformations and strains is desirable[7]. One popular method for making such measurements is Digital Image Correlation (DIC), in which a computer algorithm compares images of a sample surface collected before and after deformation to compute the displacements and strains experienced by the sample[8].

Before performing DIC, the specimen must be prepared with a high contrast speckle pattern. Ideally, a speckle pattern will be isotropic as well as non-repeating[9]. A variety of patterning methods exist. Figure 1 shows a selection of example patterns made using four different methods.

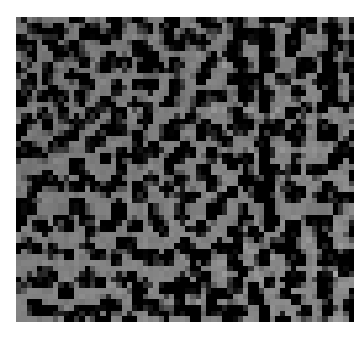

(a)

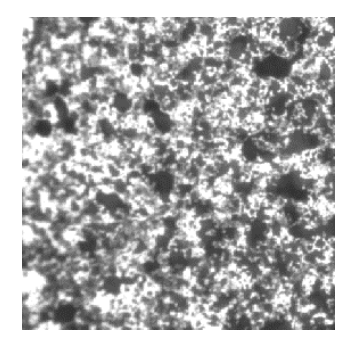

(b)

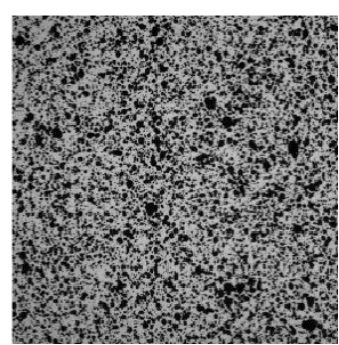

(c)

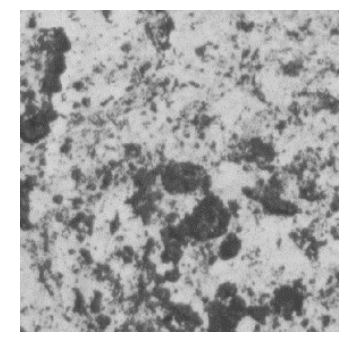

(d)

Figure 1: Example patterns for DIC created by (a) stamping, (b) splattering, (c) airbrush, and (d) sponges. 
The deformation is computed by comparing many smaller groupings of pixels within the image, called subsets. This accomplished by searching the deformed image for the subset of pixels which has the minimum squared difference in gray values when compared to a subset from the reference image[10]. Once the matching subset is identified the displacement between the two images can be calculated. This is often accomplished using the correlation function[8]:

$$
\bar{d}_{o p t}=\operatorname{argmin} \sum|G(x+\bar{d})-F(x)|^{2}
$$

Where $\bar{d}_{\text {opt }}$ is the optimal displacement vector, $\mathrm{F}$ is the reference image, and $\mathrm{G}$ is the image after displacement.

This means that each subset (SS) results in a single displacement or strain measurement. The size of the subset is limited by the size and quality of the speckle pattern used[11]. The number of pixels between each subsequent subset is called the step size (ST). In order to utilize all of the information contained in the speckle pattern the step size must be less than the size of the subset being used.

DIC holds several advantages over other methods of strain measurement: (i) that DIC is non-invasive and non-contacting to the sample (other than a thin layer of paint, which is assumed not to greatly influence deformation); (ii) the ability to obtain data for an entire area rather than a single point, and (iii) the technique can be applied to test samples over a broad range of length and time scales depending on the lenses and cameras which are used. DIC has been demonstrated at lengths ranging from nano-meters [12], [13] to tens of meters [14], and speeds ranging from quasi-static to impact [15], and is capable of making both 2D and 3D measurements[16]. 
Typically, 3D surface strain measurements using DIC require the use of at least two cameras to observe the test specimen at different perspectives, in a process referred to as 3DDIC or stereo DIC[17]. Not only is stereo DIC more expensive due to the cost of additional cameras and lenses, but the multiple cameras must be synchronized, which requires additional hardware further driving up the complexity and cost. The level of synchronization necessary for accurate stereo DIC can be time consuming and difficult to achieve[18]. In addition to the temporal calibration to ensure both cameras are taking pictures of the sample at the same time, the cameras must be calibrated spatially as well. This spatial calibration is often accomplished using a target with a grid of dots on its surface. These dots are precisely and equally spaced along the target. An example image pair of such a grating is shown in Figure 2. By capturing images of the target as it is moved in and out of plane and rotated about all three axis, it is possible to determine the intrinsic and extrinsic camera parameters as well as their positioning[19].

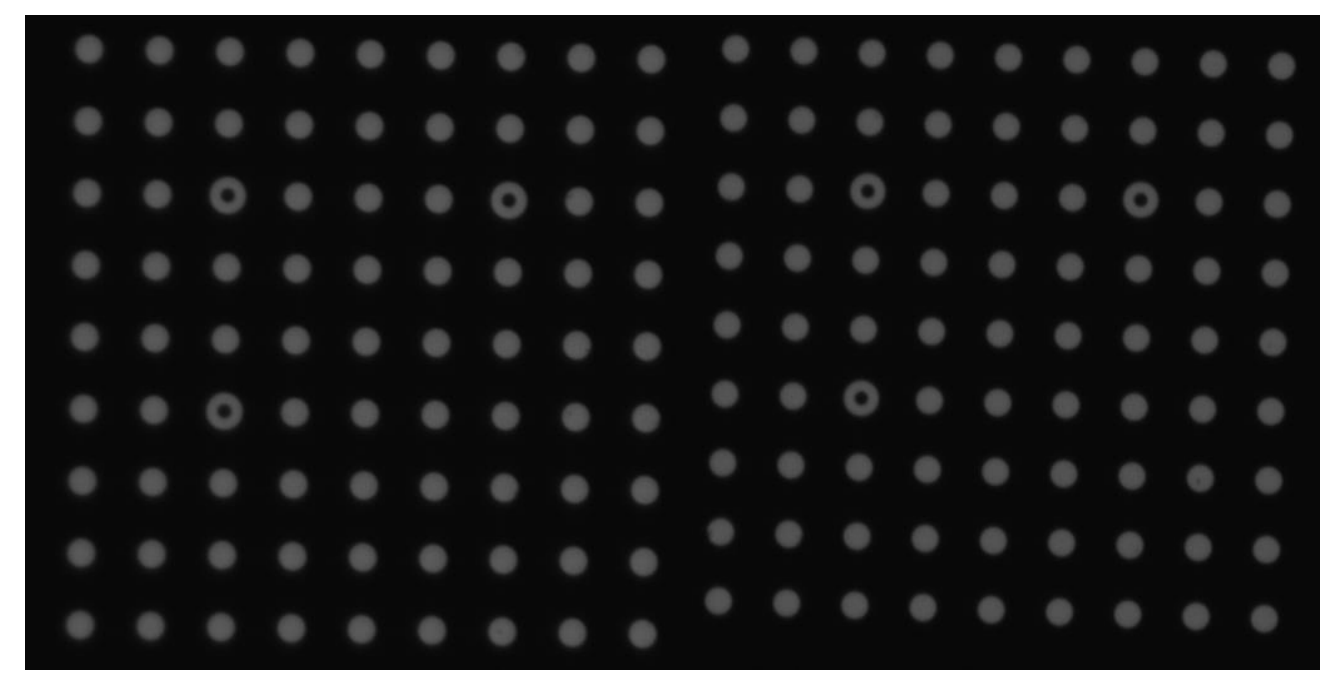

Figure 2: Views of a stereo calibration grid captured by two different cameras. 


\subsection{Diffraction of Light}

Diffraction occurs when light bends around the corners of an obstacle or slit. The reason behind why diffraction occurs lies in the way in which waves propagate. The Huygens-Fresnel principle is used to describe this propagation. This principle states that each point along a wavefront can be considered to be the center of a secondary wave[20]. Therefore, the wave-front at any later time can be considered as the sum of these various secondary waves through interference. Diffraction can be most easily observed whenever a wave encounters an obstacle or slit whose dimensions are close to the size of its wavelength. One example of this behavior is when light encounters a barrier with a slit that is close is size to the wavelength of that light (Figure 3). Multiple slits will result in interference within the diffracted light.
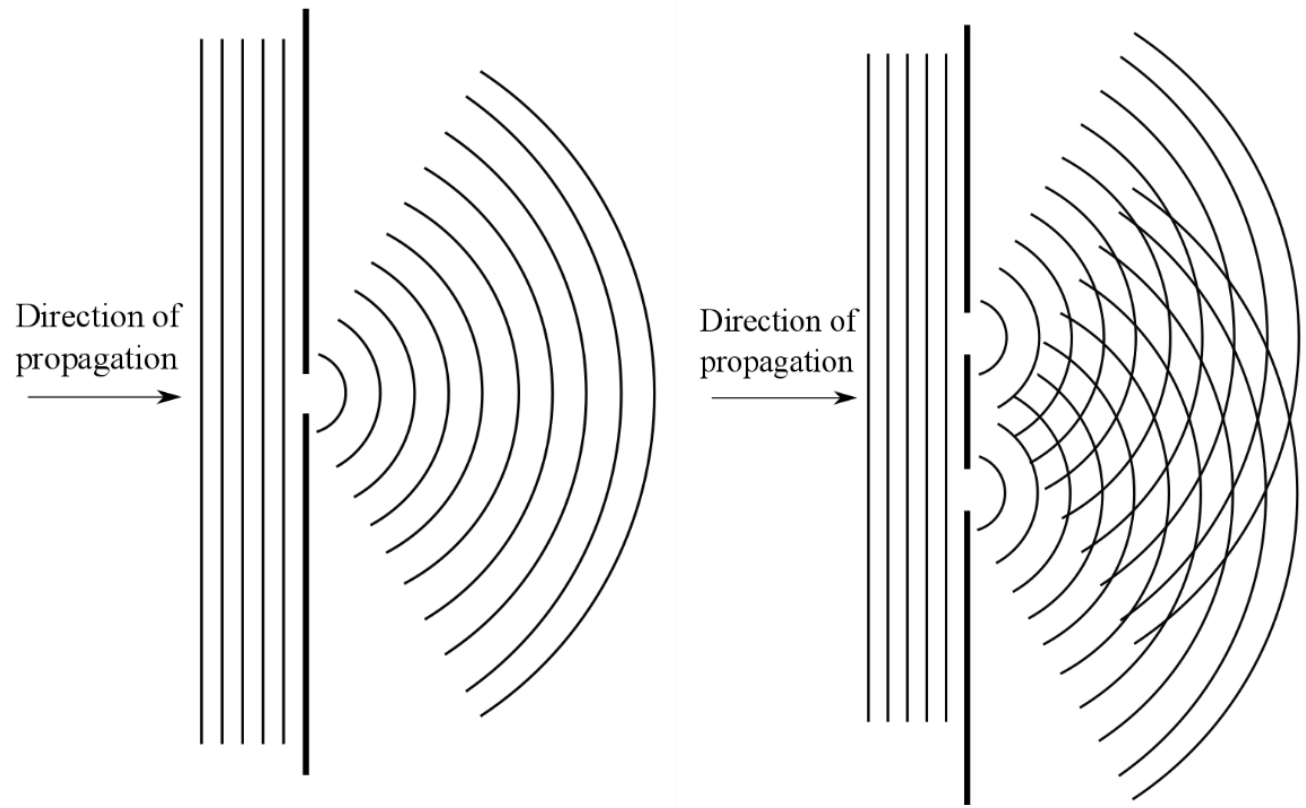

Figure 3: A representation of a wave encountering a barrier with a slit close in size to the wavelength of the wave(left) a barrier with 2 slits resulting in interference(right). 
Transmission diffraction gratings use an array of grooves to diffract light into several beams traveling in specific directions[21]. When the light diffracting from adjacent slits interfere in constructive manner different diffraction orders are formed. The center of the grating has a zero-order mode with positive and negative modes forming on either side of this mode. These various modes will have different diffraction efficiencies[22]. A blazed diffraction grating is used to preserve a single diffraction order while eliminating all others[23]. This is accomplished by changing the blaze angle $\left(\Theta_{B}\right)$ in the sawtooth profile[24]. Figure 4 shows the profile of a both a standard transmission grating and a blazed grating with rays denoting the zeroth and positive and negative first orders.

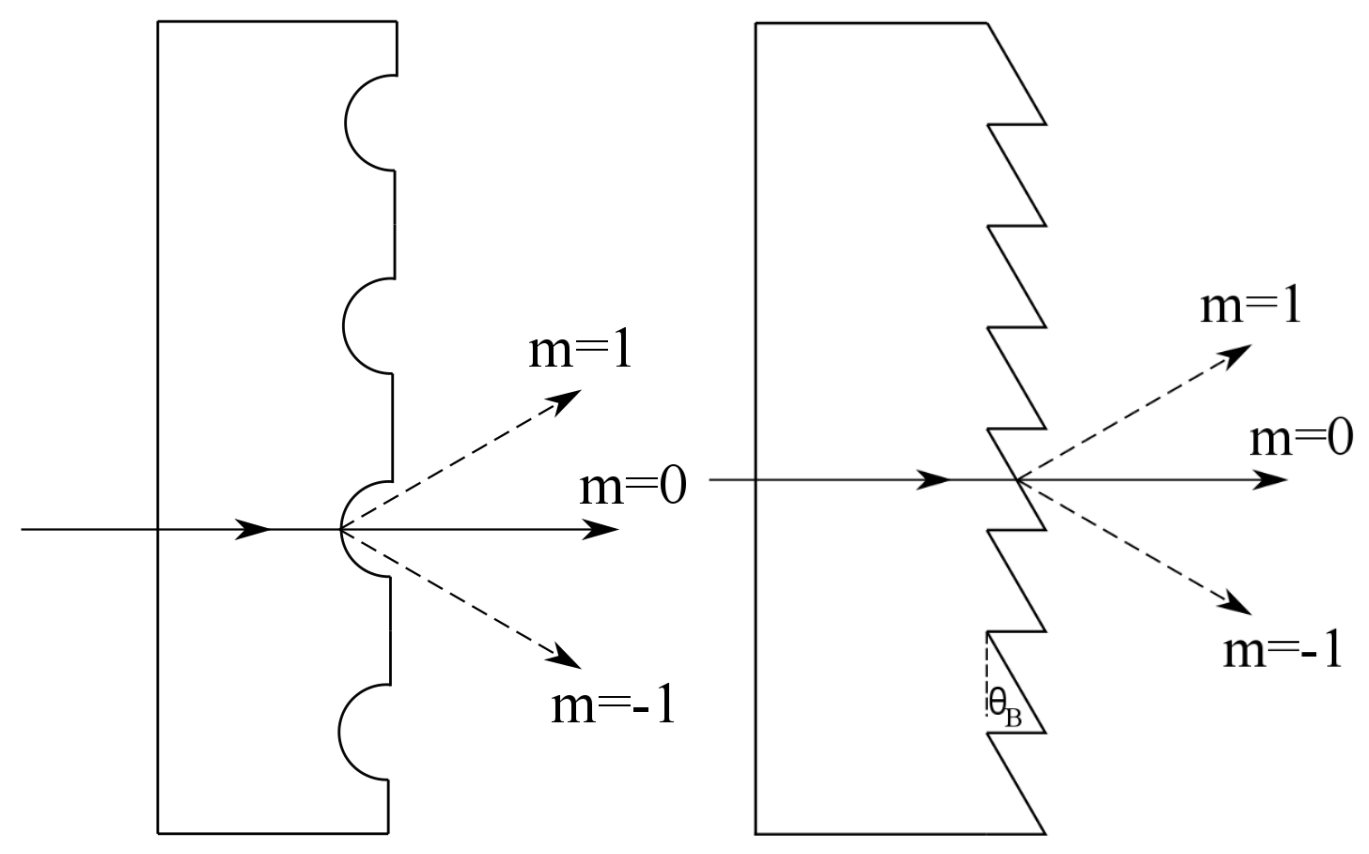

(a)

(b)

Figure 4: Typical groove geometry for standard transmission grating (a) and blazed grating (b) $m$ denotes the diffraction order. $\theta_{B}$ is the blaze angle. 


\subsection{Diffraction Assisted Image Correlation}

Recently, Xia et al. developed a technique to enable 3D-DIC measurements to be made using a single camera which they refer to as Diffraction Assisted Digital Image Correlation (DAIC)[25]. The principle behind how DAIC works is demonstrated by the ray diagram in Figure 5. In DAIC, the single camera is augmented by placing transmission diffraction gratings at a precise distance between the camera and sample. When the light reflected off the sample interacts with the grating, the rays bend before reaching the camera sensor. The bent rays result in higherorder diffracted images, corresponding to the different diffraction orders. These images can be considered the result of virtual objects which are perceived by the sensor and are labeled + and

- in Figure 5. This work focused on the positive and negative first-order images. The angle of this first order image depends on the wavelength of the light source and the pitch of the grating. This relationship is characterized by the following equation:

$\theta=\sin ^{-1}(\lambda / \mathrm{p})$

Where $\lambda$ is the wavelength of the source light and $p$ is the pitch of the grating (1/Line Density). 


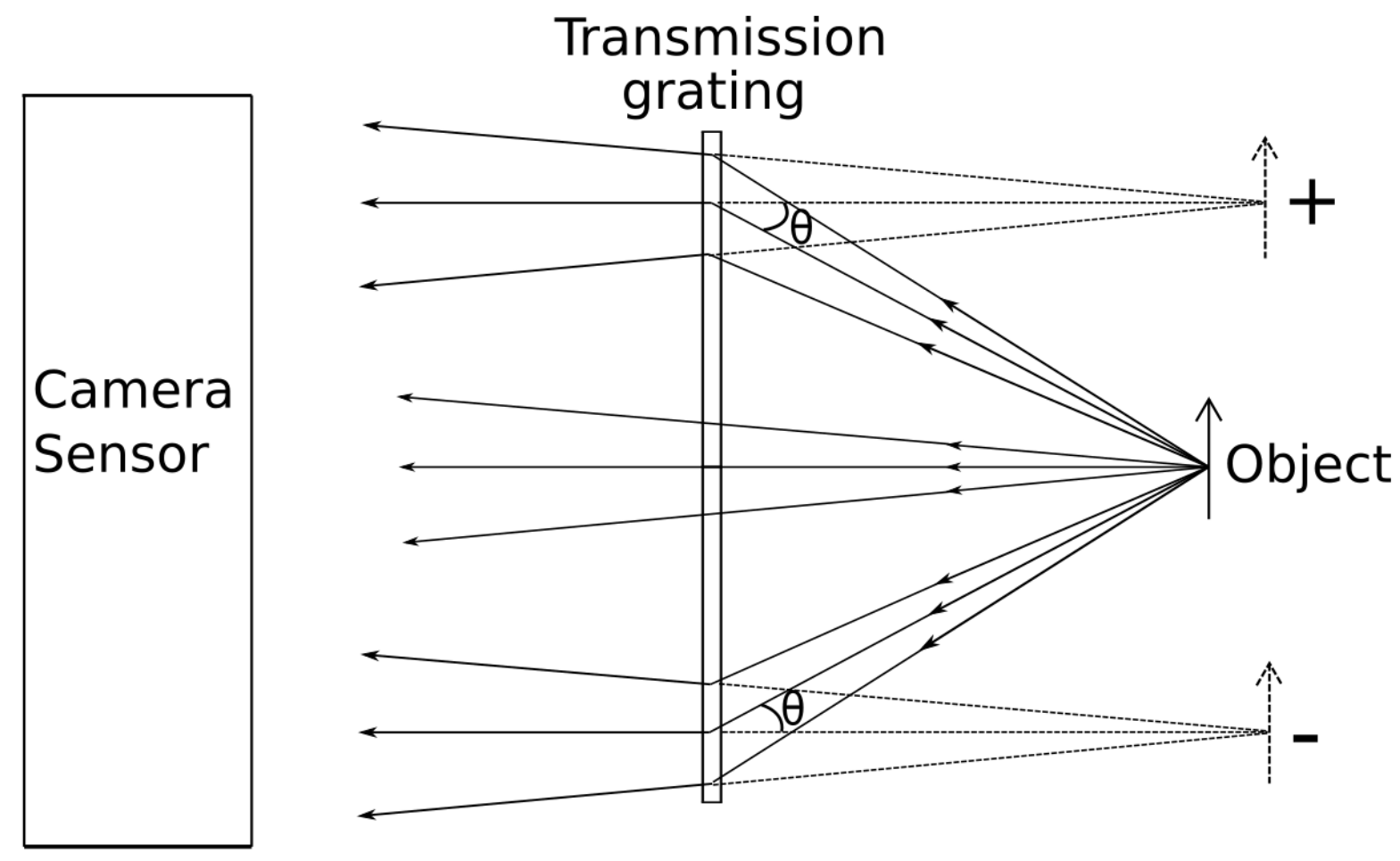

Figure 5: Optical arrangement for use of DAIC to observe two stereo perspectives with a single camera

The higher-order images provide multiple views of the sample[26] to be captured by the camera and analyzed to measure 3D displacements and strains[27]. These first order images can also be analyzed to provide measurement of 3D surface profiles [28]. DAIC was demonstrated at room temperature using monochromatic red light, but in principle the technique can be used for any wavelength of monochromatic light.

\subsection{High Temperature DIC}

When performing DIC at high temperature several difficulties can arise due to the elevated temperature[29]. Care must be taken to ensure the paint used to make the speckle pattern can survive whatever temperatures the specimen may experience. This can be 
accomplished through the use of paints and coatings designed to retain color as well as contact with the specimen at high temperatures[30]. This insures that the speckle will remain in place while providing the necessary contrast. For tests performed in ambient air, the challenge of heat haze occurring between the camera and the sensor must be addressed. One solution which has been presented is the use of an air knife to mix the air[30].

Another challenge is the fact that, at high temperatures, materials emit light which has a much higher intensity at longer wavelengths (including red) than at shorter wavelengths (such as ultraviolet). If the temperature gets high enough, the emitted light can saturate a camera's sensors making the images captured unsuitable for use in DIC. It has been shown that by using an ultraviolet (UV) bandpass filter, DIC can be performed at much higher temperatures before the emitted light has a significant impact on the image quality[31]. In this study, DAIC is performed with monochromatic UV light instead of red light, demonstrating that DAIC can not only perform stereo imaging using a single camera, but can do so at extreme temperatures. The combined UV-DAIC technique offers a cheap and simple way to measure 3D deformation and strain at high temperature.

\subsection{Virtual Strain Gauge Study}

One question faced by anyone using DIC is determining which settings, such as subset step size and strain window, will yield the best results. One process which can help in this determination is a Virtual Strain Gage (VSG) analysis following a procedure set by Phillip Reu[32]. The goal of a VSG study is to determine the best balance between filtering and noise. Under the VSG procedure, three images are compared using DIC: the reference image, an unloaded "noise" image, and a maximum deformation image. The noise image is used to calculate the standard 
deviation of the strain throughout the image, which is used as an indicator of the precision of the measurement. The maximum deformation image is used to determine the peak strain in the image, which is compared against a known expected value as an indicator of accuracy. The procedure is repeated for various combinations of subset size, step size, and strain window. The VSG and Spatial Resolution (SR) are calculated using the following equations:

$V S G=((S W-1) S T)+1$

$S R=((S W-1) S T)+S S$

It can be seen from these equations that the VSG is the number of pixels between the centers of the leftmost and rightmost subsets used to calculated strain, while the spatial resolution is the number of pixels from the leftmost edge of the leftmost subset to the rightmost edge of the rightmost subset (i.e. the shortest distance between two non-overlapping strain measurements). Figure 6 shows these measurements as well as subset size and step size using an example array of subsets used to calculated strain. The VSG size gives an indication of the area over which the strain measurements have been averaged[33]. A large VSG will result in a smooth contour at the cost of a loss in spatial resolution. The final step is to extract the strain data along a line which contains high strain gradients and plot the results found using the various combinations of settings. These strain contours are checked for convergence with the optimal setting being those with the smallest VSG while still converging. For the purposes of this study a contour is said to converge when the contour can be matched using larger VSG sizes. 


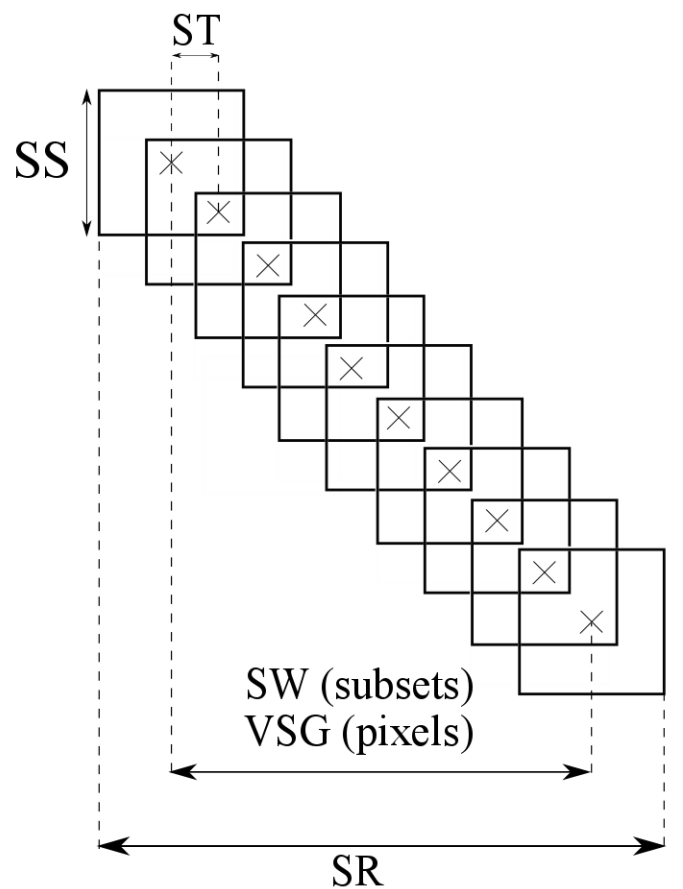

Figure 6: An array of subsets used to make a strain measurement. The center of each subset is marked with an $x$. 


\section{CHAPTER 2}

\section{OBJECTIVES}

- $\quad$ Adapt the current technique for performing DAIC for use with UV light.

- Validate this new experimental method at room temperature using rigid body motion.

- Perform UV-DAIC on a specimen at an extreme temperature and record displacement and strain data.

- Perform a Virtual Strain Gauge analysis to determine the optimal DIC settings. 
CHAPTER 3

APPROACH

\subsection{Experimental Set Up}

Tests were performed using thin, rectangular samples of Hastelloy- $\mathrm{X}$ which have dimensions of 1 inch wide, 6 inches long, and 0.025 inches thick. The sample was clamped at the bottom with the sides and top left free. A random, black and white speckle pattern was applied to one side of the sample using VHT Flame-proof high temperature paint and a stamp rolling kit from Correlated Solutions Inc. An example photo such a specimen and its surrounding test fixtures is shown in Figure 7, while the broader experimental setup is shown in Figure 8. The specimen was heated from behind via magnetic induction using a coil made of 3/16 inch diameter soft copper tubing (not visible in Figure 7, but shown hovering behind the sample in Figure 8). This method of heating was selected to afford a non-contact method which generates heterogeneous temperature distributions. The specimen was flanked by two silicon carbide plates to provide a uniform black background upon which to project first order diffraction images, while concealing the induction coil from appearing in the images. Temperature was monitored using a FLIR A6751sc infrared (IR) camera. No significant heating was observed in the silicon carbide due to induction heating. 


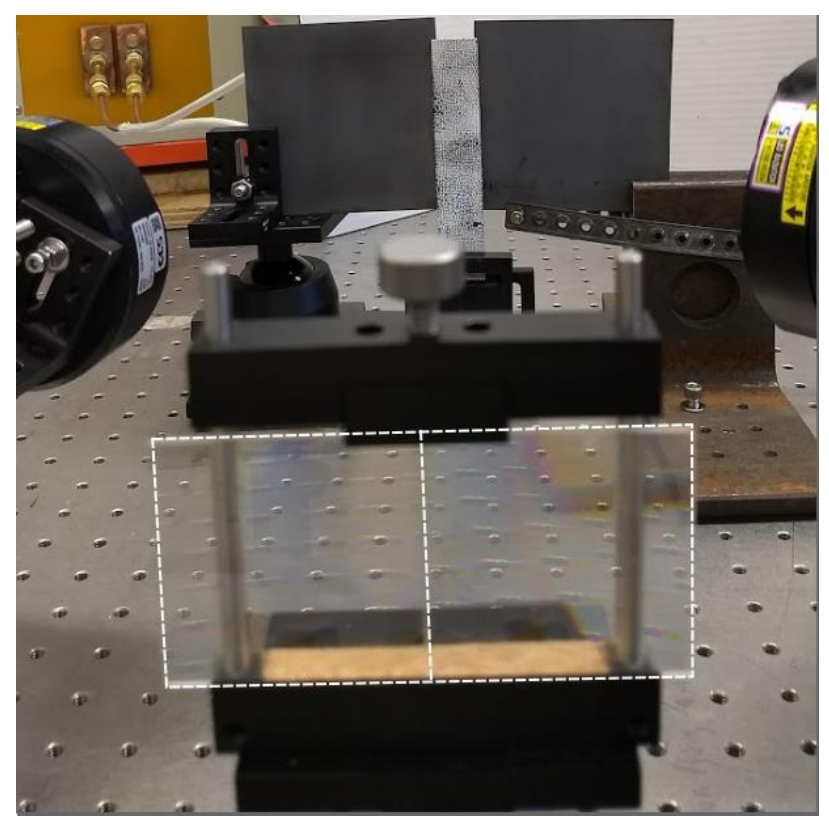

Figure 7: Front view of experimental set up with two diffraction gratings outlined by dashed line

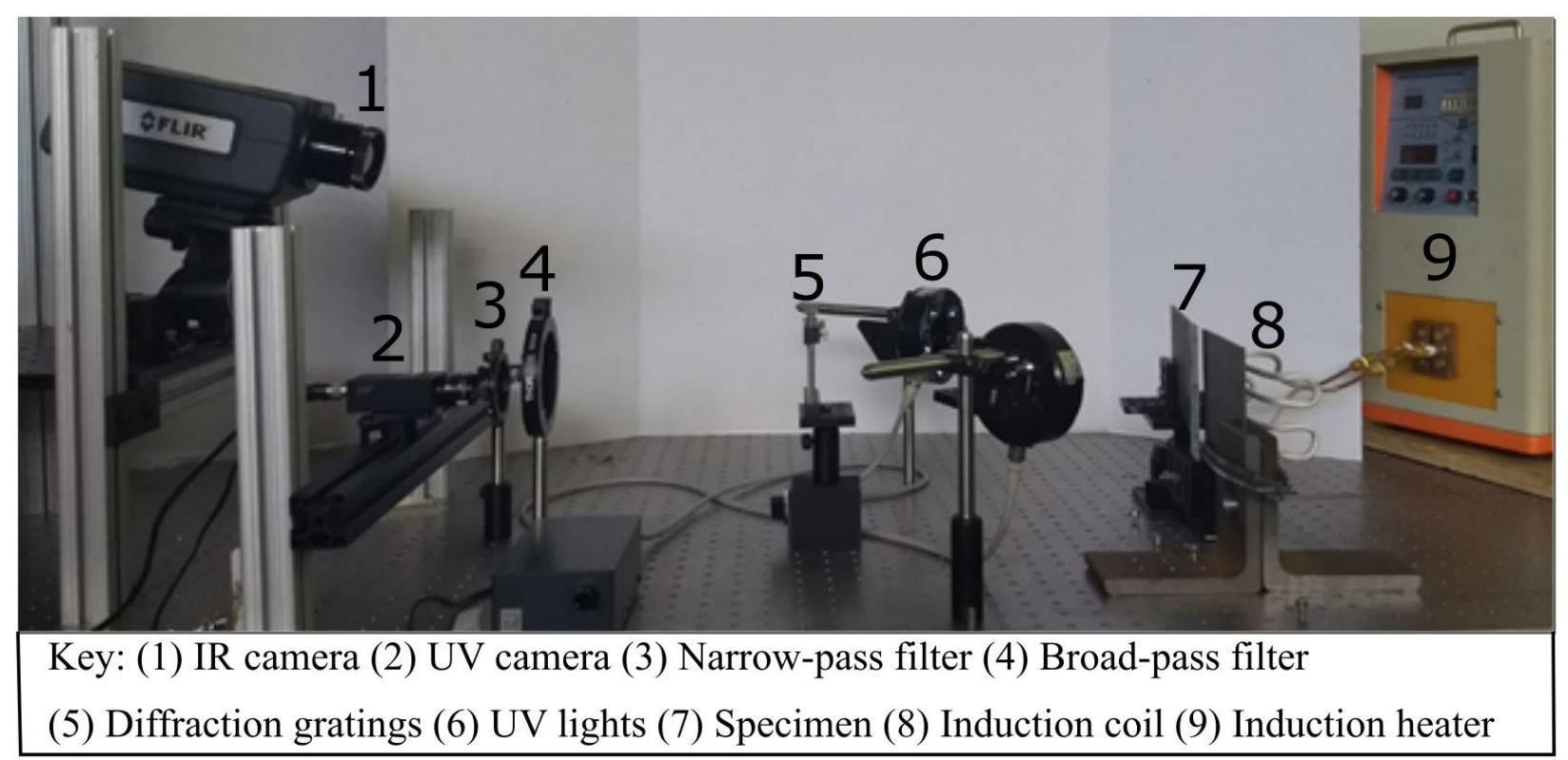

Figure 8: Side view of experimental set up 
UV images were captured using a single JAI CM-140GE-UV camera equipped with a 25 mm lens from Universe Kogaku Optics (model uv25-0327). The camera was placed a distance of 28 inches away from the specimen. A matching set of two blazed transmission gratings with a groove density of 300 grooves/mm (Edmund Optics part \#85-296) were placed between the camera and the specimen at a distance of 15 inches away from the specimen, such that the first order image produced by each grating will not overlap with the zeroth order image. These gratings are outlined by a dashed white line in Figure 7. Illumination was provided by two LED light sources with a peak wavelength of $365 \mathrm{~nm}$. Two UV-range optical bandpass filters were applied in series; a "broad" filter with a full-width at half-max (FWHM) bandwidth of $80 \mathrm{~nm}$ (XNite 330C from LDP LLC), and a "narrow" filter with a FWHM of 2 nm (MaxLine Laser Line Filter 364 from SemRock). Both filters allow a high level of transmission at wavelengths of $365 \mathrm{~nm}$. The broad filter has been demonstrated to enable UV-DIC on Hastelloy-X to temperatures of at least $1125^{\circ} \mathrm{C}[31]$, but its bandwidth is too broad to pair with the diffraction gratings. The narrow filter produces sharper images when paired with the gratings but transmits significant red and infrared wavelengths which are undesirable at high temperature. The transmission curves of the optics are shown in Figure 9. 


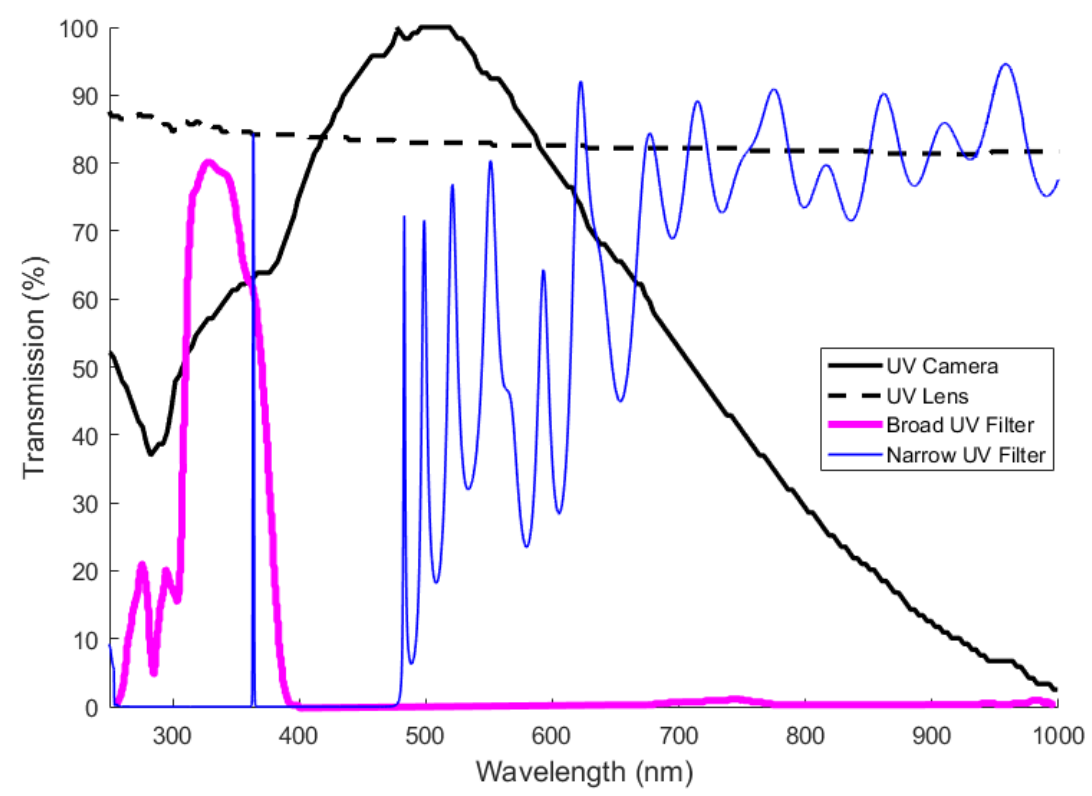

Figure 9: Transmission curves for UV optics and camera used in the experiment

\subsection{Room Temperature Experiment}

Experiments were performed first at room temperature, then at high temperature. In the room temperature experiments, a static "reference" image was collected, followed immediately by a "noise" image with no motion in between. This "noise" image is used to determine the resolution of the measurement. A series of known in-plane displacements were then applied to the sample using a micrometer driven translation stage, with additional images captured after each motion. The specimen was moved a total of 0.2 inches in 0.01 inch increments. The images captured before and after this motion were then used to compute displacements using stereo

DIC. The procedure was then repeated for out-of-plane translations ranging from 0.01 to 0.2 inches at intervals of 0.01 inches. An example of the images captured of the specimen is shown in Figure 10. 


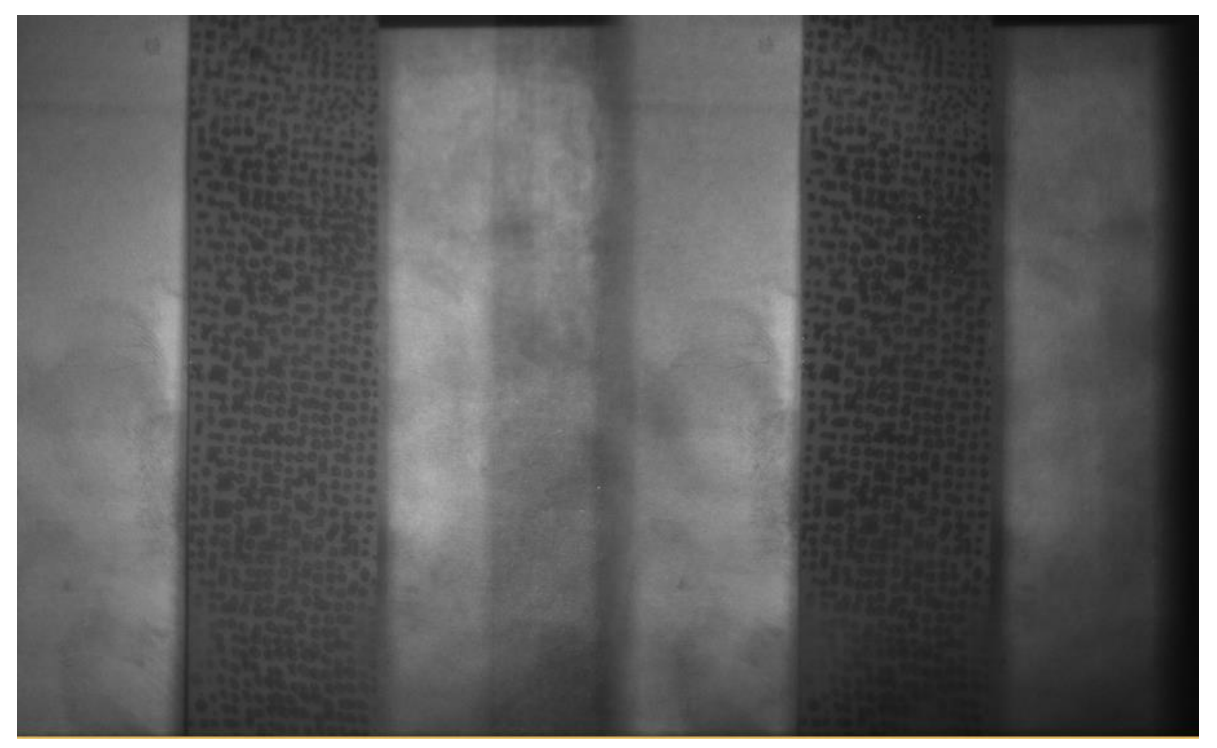

Figure 10: An example of the two first order images captured using a pair of blazed gratings and a single camera. The zeroth order image is barely visible in the center of the image.

\subsection{High Temperature Experiment}

Next an experiment was performed at high temperature. The specimen was heated using magnetic induction using the coil shown in Figure 8. The coil was placed behind the silicon carbide plates to prevent the edges of the coil from appearing in the image, thus interfering with the higher order images produced by the gratings. A new reference image was captured at room temperature, then UV and IR images were periodically captured as the sample is heated to 900 ${ }^{\circ} \mathrm{C}$.

Figure 11 shows an image of the highest temperature profile captured by the IR camera. The camera used has a resolution of $640 \times 512$ pixels and a framerate of 125 frames per second. The temperature profile is highly non-uniform, with temperatures ranging from below $350{ }^{\circ} \mathrm{C}$ to above $900{ }^{\circ} \mathrm{C}$. At these high temperatures, the IR camera requires a neutral density filter as part 
of its calibration, so temperatures below $350{ }^{\circ} \mathrm{C}$ are indistinguishable from the ambient temperature of the room. To better visualize the specimen in the figure, a white dashed line has been added to outline the edges of the specimen.

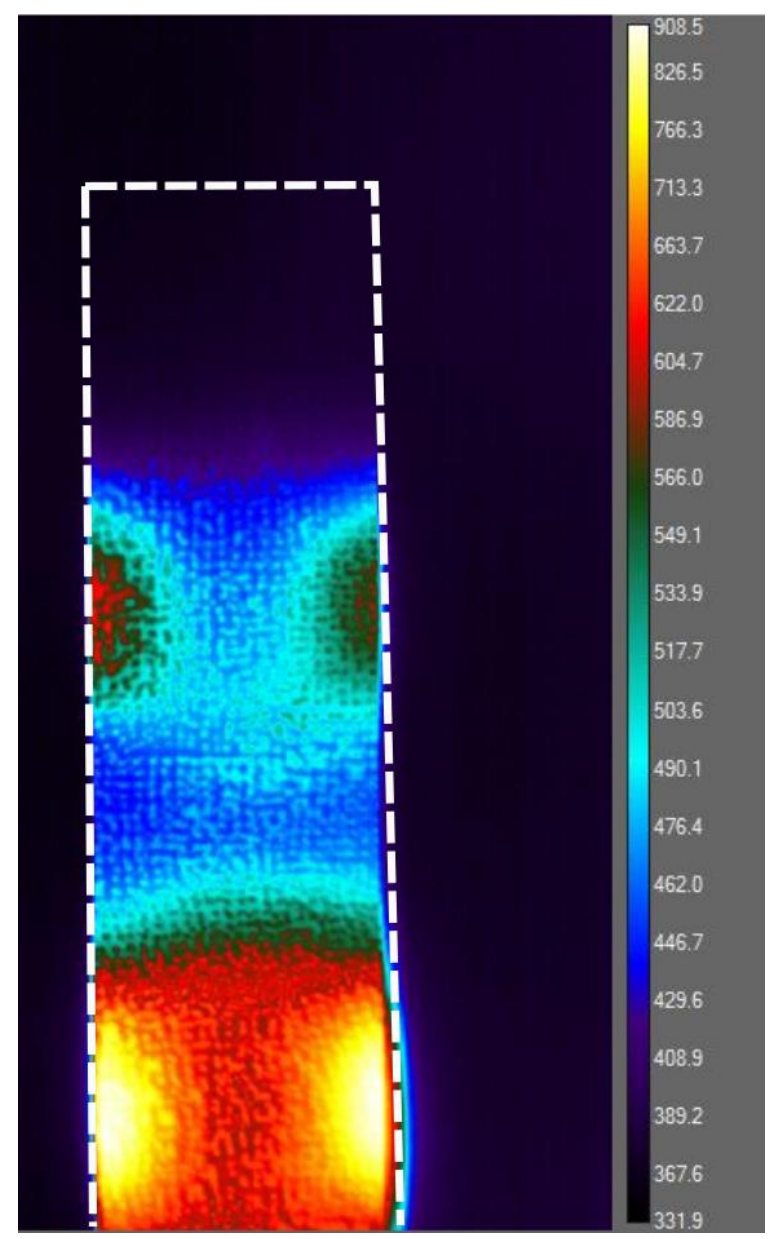

Figure 11: Temperature Profile in ${ }^{\circ} \mathrm{C}$ captured using IR camera, the outline of the specimen is shown by the dashed line 


\subsection{Post Processing}

For both the room temperature and high temperature test, some minor post processing was necessary before the captured images could be used for DIC. The DIC software used for these experiments (VIC-3D by Correlated Solutions Inc.) requires a separate image file for each image in the pair used for measurement. For this reason, each raw image needed to be split into a left and right half to form two new image files, with each half containing one of the first order images created by the diffraction gratings. These split images were used with the VIC-3D software to calculate deformation and strain using the optimal subset size (SS), step size (ST), and strain window (SW as determined from a VSG study). The DIC parameters are summarized in Table 1. The values for virtual strain gauge and spatial resolution were found using equations from Reu [32].

Table1: Parameters used to perform stereo DIC.

\begin{tabular}{|l|l|}
\hline Camera Type & JAI CM-140GE-UV \\
\hline Image Size & $1392 \times 1040$ (pixels) \\
\hline Frame Rate & 16 frames/second \\
\hline Lens & Universe Kogaku Optics UV2528B \\
\hline DIC Software & Vic-3D v8 \\
\hline Scaling & $0.1176 \mathrm{~mm} /$ pixel \\
\hline Subset Size & $41 \times 41$ pixels \\
\hline Step Size & 15 pixels \\
\hline Strain Window & 23 subsets \\
\hline Virtual Strain Gauge & 333 pixels \\
\hline Spatial Resolution & 371 pixels \\
\hline Correlation Algorithm & Zero-normalized square difference \\
\hline Interpolation & Optimized 8-tap \\
\hline
\end{tabular}


CHAPTER 4

RESULTS

\subsection{Room Temperature Results}

The average in-plane displacement over the entire region of interest is plotted against the applied horizontal displacement in Figure 12(a), while the average out-of-plane displacements are plotted in Figure 12(b). The horizontal axis represents the known displacement applied by the micrometer to the sample while the vertical axis represents the average measured displacement acquired using UV-DAIC. Each data point also includes uncertainty bands depicting one standard deviation of the measured motion. The bands in part (a) are so narrow they appear to be flat lines. The plots include a 45 degree dashed line which represents perfect agreement between the applied and measured displacement. In each case, the dashed line is well within the uncertainty bands for each measurement. Parts (c) and (d) of the figure show the percent error of the measurements in parts $(\mathrm{a})$ and $(\mathrm{b})$ respectively. 


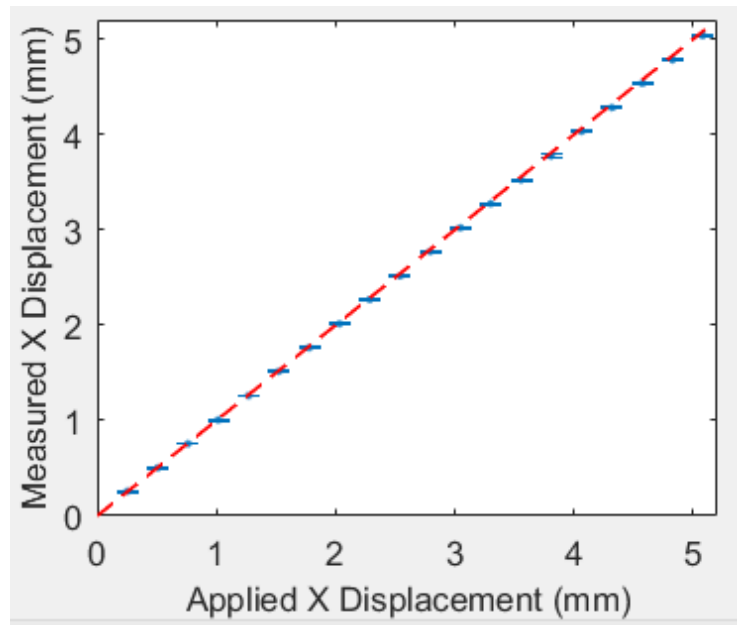

(a)

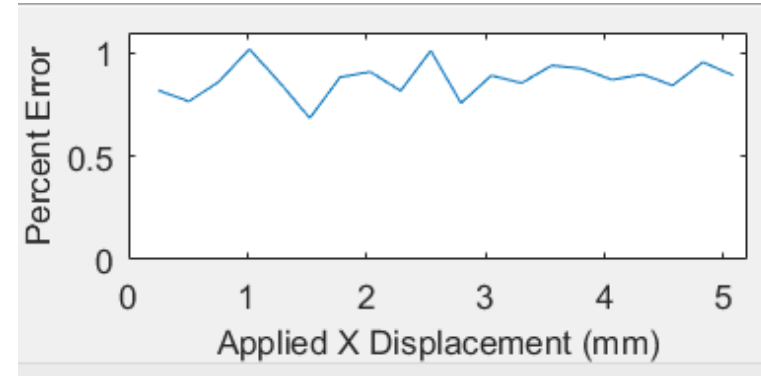

(c)

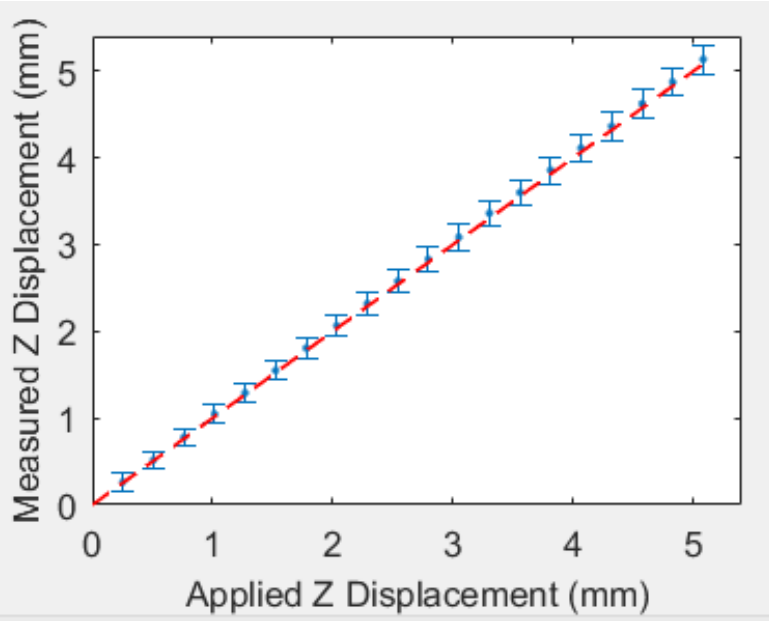

(b)

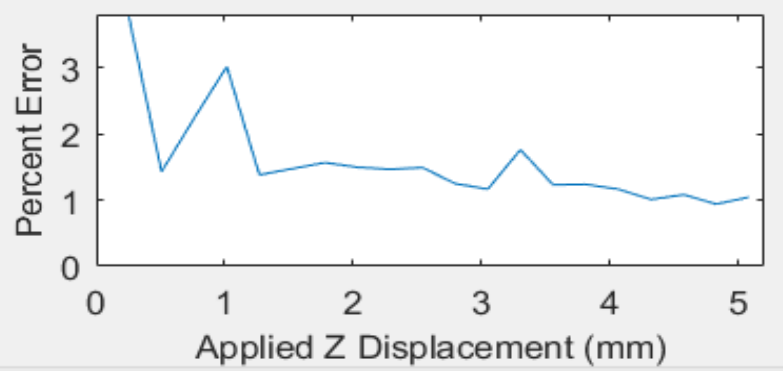

(d)

In-plane

out-of-plane

Figure 12: Plot of measured vs applied displacements as well as the percent error between these displacements in the in-plane direction $(X)(a)$ and $(c)$ as well as the out-of-plane direction (Z) (b) and (d).The dashed red line represents perfect agreement between the applied and measured displacement. Each measurement is the mean value for the entire region of interest, with uncertainty bands showing two standard deviations.

\subsection{High Temperature Results}

Under the applied temperatures in Figure 11, the in-plane normal strains are plotted in Figure 13, while the out-of-plane displacements are plotted in Figure 14. The plots in both figures are superimposed over the left image of the deformed sample. As the temperature increased over the course of the experiment the free end of the sample, which was farthest from the 
clamped base, bent toward the camera. This behavior was captured very well by the out-of-plane displacement measurements.
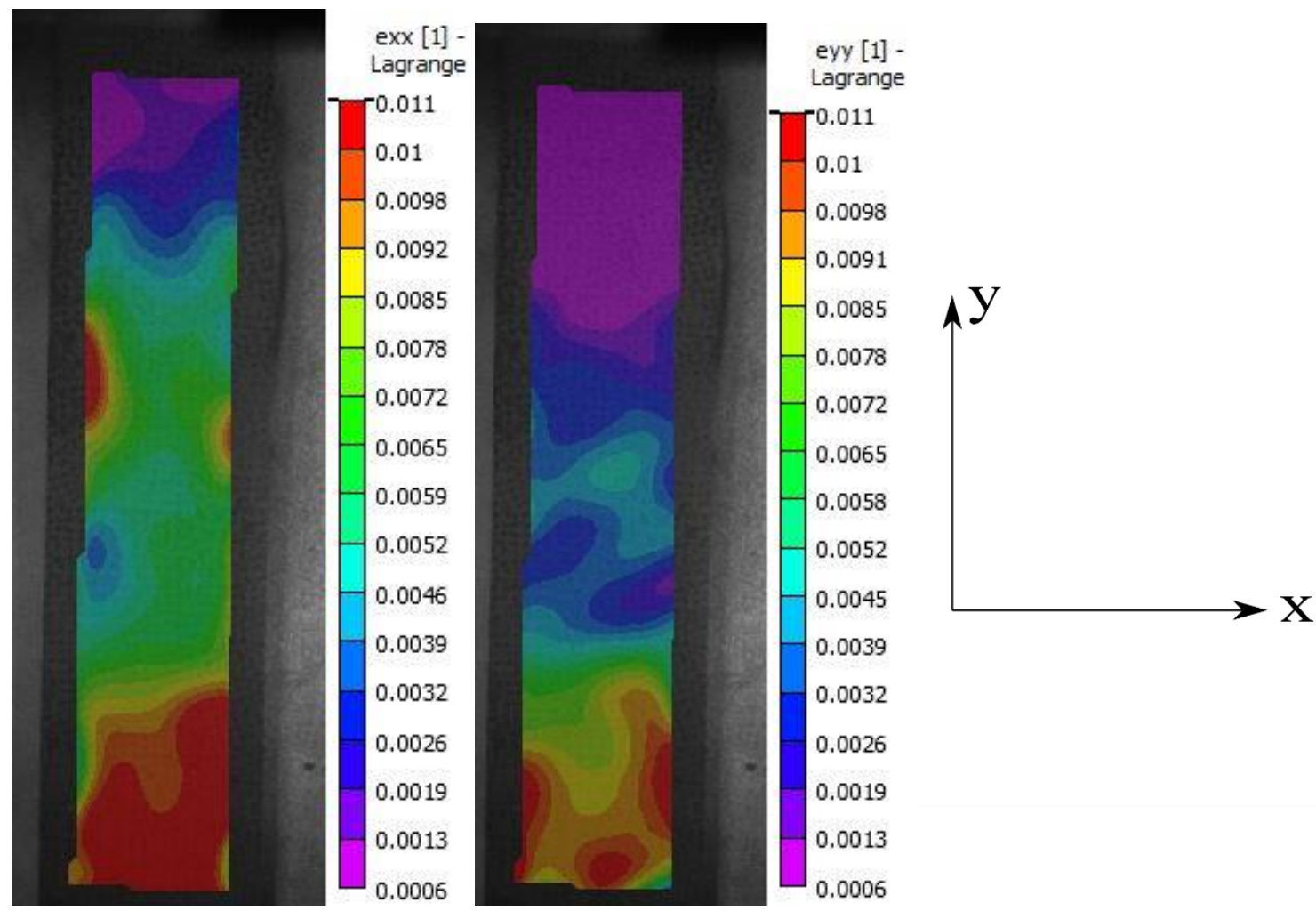

Figure 13: The normal strain field in both the $x$ and $y$ direction at the temperature distribution in

Figure 11. 


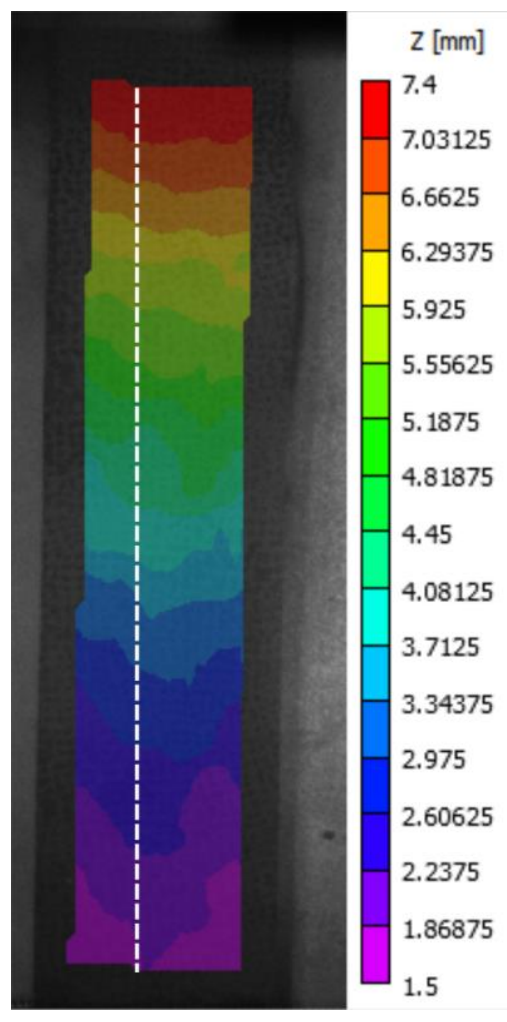

(a)

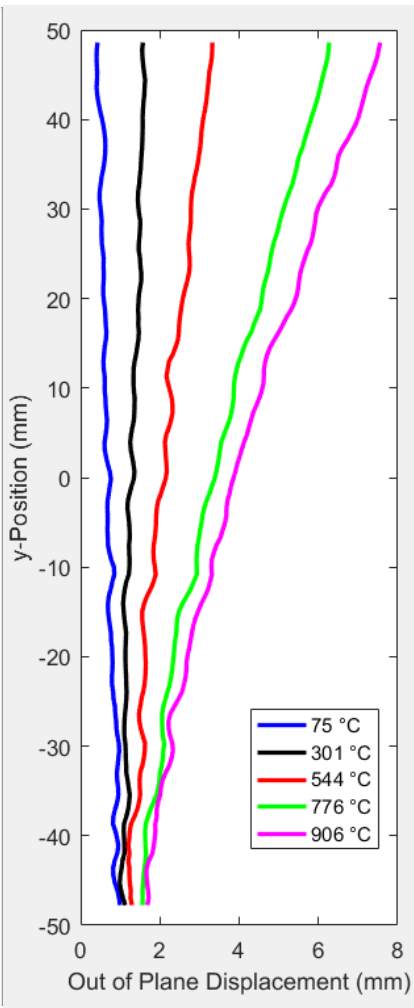

(b)

Figure 14: The out of plane displacement of the specimen at the temperature shown in Figure 11 (a); and a plot of the out of plane displacement of the specimen along the dashed white line as temperature increased (b). The temperatures given in the legend are the peak temperatures recorded in the specimen.

By comparing the two strain contours it can be seen that highest strain was measured in the bottom fourth sample where the temperatures were highest, while lower strains were measured in the upper portion where temperatures of the were lower. These results are consistent with the strains one would expect under thermal expansion.

\subsection{Virtual Strain Gauge Results}

In order to display the results of the VSG study, the strain values which were calculated using different DIC settings are plotted together to form the plot shown in Figure 15b. The relative 
gauge size (VSG) for each setting also appears on the left side of the plot. This strain data was taken from the line shown in Figure 15a with each setting. This line was chosen so that it has a high strain gradient in order to better compare the results. The optimal settings were determined by finding which contour has the smallest gauge size while still converging. For this test the optimal settings were chosen as a subset size of 41 pixels, a step size of 15 pixels and a strain window of 23 subsets.

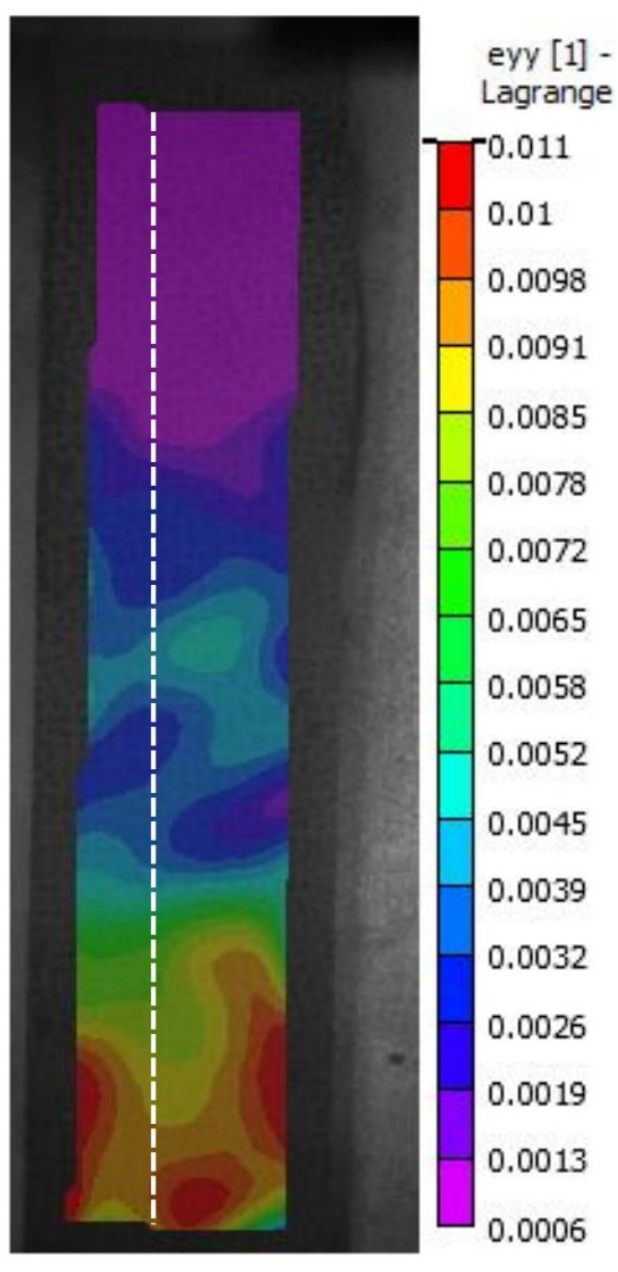

(a)

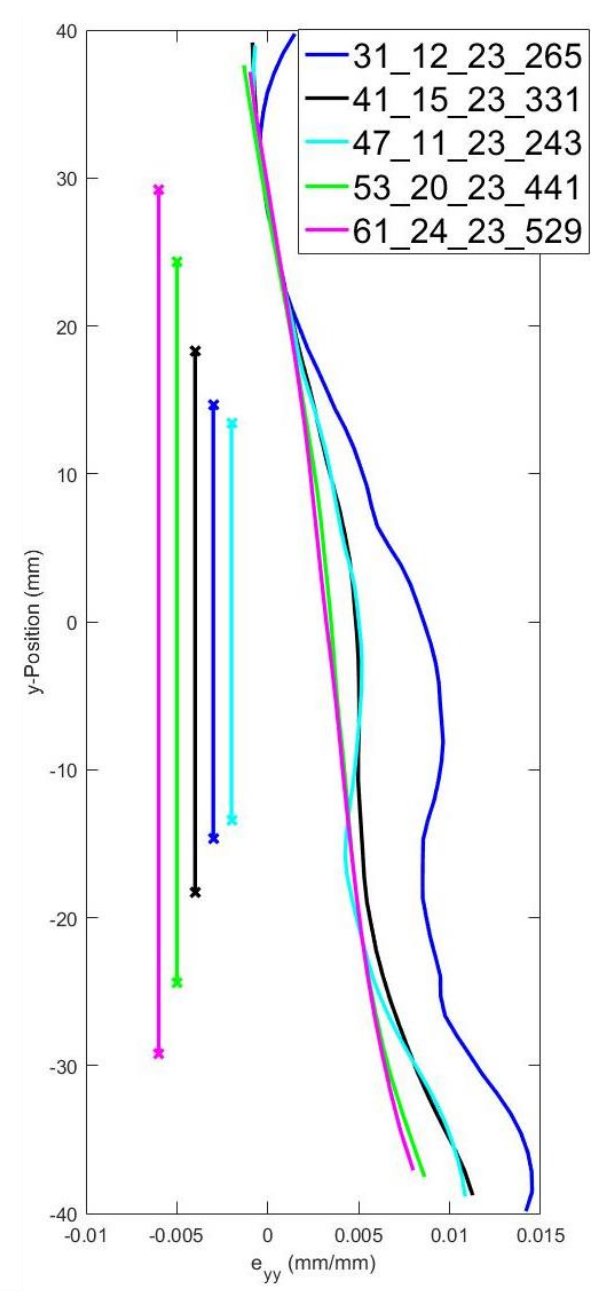

(b)

Figure 15: A plot of the strain in the y direction with the position of the line scan shown (a) Strain calculated along this line using different DIC settings (b). The legend shows the settings in the format SS_ST_SW_VSG. The relative gauge size for each result is shown on the left side 


\section{CHAPTER 5 \\ DISCUSSION}

\subsection{Effect of UV Filters on Calibration}

The results of Figures 12-14 show that UV-DAIC was successfully demonstrated at room temperature and high temperature. However, the quality of the UV-diffracted images is highly dependent on the bandwidth of the UV filter. Figure 16 shows the difference in image quality using images of the stereo calibration grid used by VIC-3D to calibrate the stereo cameras. The left image was captured using only the broad pass filter, the center image was captured using only the narrow filter, and the right image was captured using both filters in series. The left image in Figure 16 used an exposure time of $5890 \mu$ s while the center and right images both used an exposure time of $31,572 \mu \mathrm{s}$. This exposure time was selected in order to ensure enough light reached the sensor through the series of filters while also minimizing aperture in order to maximize depth of field. No other camera settings were altered between the images. Because the angle at which images produced by gratings depends on the wavelength of light, the use of a single broad pass filter resulted in the camera capturing several overlapping first order images corresponding to different wavelengths of light, resulting in blurry images that could not be calibrated. When replaced by the narrow filter with a full width at half maximum (FWHM) of 2.5 $\mathrm{nm}$ a much clearer image was captured, resulting in images clear enough for calibration and correlation at room temperature. However, the narrow filter transmitted significant levels of infrared light, making it unsuitable by itself for high temperature measurements. Only when the 
two filters are combined in series does it become possible to get crisp UV images which remain minimally saturated at high temperature.

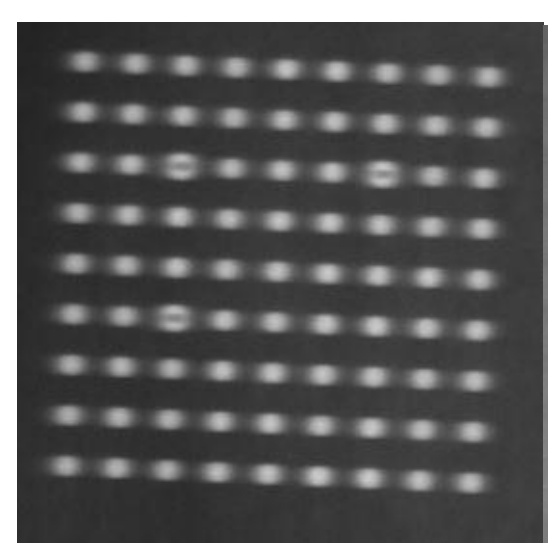

(a)

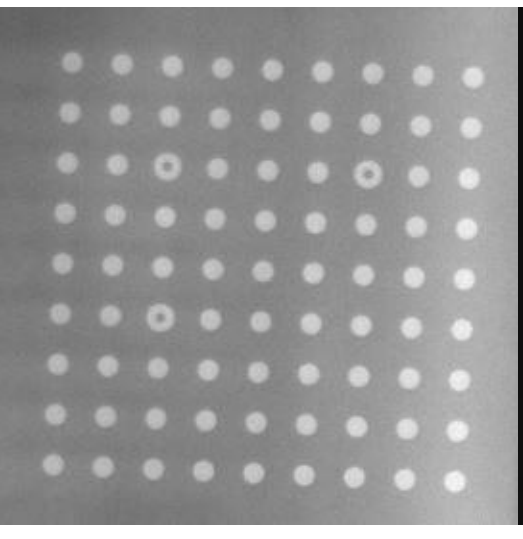

(b)

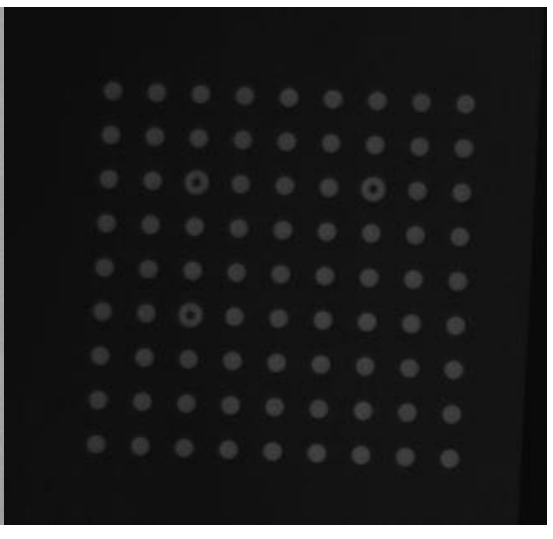

(c)

Figure 16: Images of calibration grid with various filters. Single broad pass (a), Single narrow filter (b), both in series (c)

\subsection{Optimization of Available Pixels}

The transmission gratings used in this work are blazed transmission gratings, which have the property that the majority of the light is concentrated to the zeroth and positive first order images, effectively eliminating second and higher order images. In order to avoid overlap between the zeroth-order images and first-order images, only a fraction of the camera's available pixels were able to be used. This means that the width of each image has an upper limit of one third of the total camera's pixels in the direction of the diffraction. In our experiment this is the horizontal direction. One method to improve the utilization of pixels proposed by Xia et. al. is to use a custom made transmission grating with suppressed zeroth-order intensity[25]. Through specific groove profile design it is possible to create a grating where the zeroth order 
image is suppressed[34]. With the zeroth order image thus removed, both first order images can appear next to each other, providing a much more efficient use of pixels.

However, the design and production of custom gratings is complicated and more expensive than using the gratings readily available commercially. For this reason, DAIC works best in experiments which employ long, narrow specimens which do not otherwise need the full width of the image, as is the case in this experiment. As the diffraction gratings only limit the use of pixels in the horizontal direction, there is effectively no such limit on the available pixels in the vertical direction. This means that this method is especially effective when used on specimens that for specimens which already have a long slender shape rather than a more compact specimen. 


\section{CHAPTER 6}

\section{CONCLUSION}

UV-DAIC is a method which combines UV-DIC with DAIC to harness the unique benefits of each. In UV-DIC, UV lights and filters are used to screen out light emitted by specimens at high temperature, thus extending the temperature range in which DIC can be performed. In DAIC, two diffraction gratings are used to perform stereo-DIC using a single camera. In this work, rigid body motion measurements at room temperature show good agreement between the applied motion and the measured displacement in both in-plane and out-of-plane directions. At high temperature, UV-DAIC correctly detected the out-of-plane bending motion of the beam-like specimen under non-uniform temperatures, and the resulting strain fields showed the appropriate increased strain in areas with higher temperature. UV-DAIC has been demonstrated to temperatures of at least $900^{\circ} \mathrm{C}$, and could potentially be implemented at much higher temperatures if sufficient heat and light sources are used. 


\section{CHAPTER 7}

\section{REFERENCES}

[1] F. Monteverde and R. Savino, "Stability of ultra-high-temperature ZrB2-SiC ceramics under simulated atmospheric re-entry conditions," J. Eur. Ceram. Soc., vol. 27, no. 16, pp. 47974805, Jan. 2007.

[2] K. Daryabeigi, "Thermal Analysis and Design Optimization of Multilayer Insulation for Reentry Aerodynamic Heating," J. Spacecr. Rockets, vol. 39, no. 4, pp. 509-514, 2002.

[3] M. M. Opeka, I. G. Talmy, and J. A. Zaykoski, "Oxidation-based materials selection for $2000^{\circ} \mathrm{C}+$ hypersonic aerosurfaces: Theoretical considerations and historical experience," J. Mater. Sci., vol. 39, no. 19, pp. 5887-5904, Oct. 2004.

[4] D. M. V. Wie, D. G. Drewry, D. E. King, and C. M. Hudson, "The hypersonic environment: Required operating conditions and design challenges," J. Mater. Sci., vol. 39, no. 19, pp. 5915-5924, Oct. 2004.

[5] C. W. Forsberg, "Hydrogen, nuclear energy, and the advanced high-temperature reactor," Int. J. Hydrog. Energy, vol. 28, no. 10, pp. 1073-1081, Oct. 2003.

[6] K. L. Murty and I. Charit, "Structural materials for Gen-IV nuclear reactors: Challenges and opportunities," J. Nucl. Mater., vol. 383, no. 1, pp. 189-195, Dec. 2008.

[7] B. Pan, D. Wu, Z. Wang, and Y. Xia, "High-temperature digital image correlation method for full-field deformation measurement at $1200{ }^{\circ} \mathrm{C}$," Meas. Sci. Technol., vol. 22, no. 1, p. 015701, 2011.

[8] M. A. Sutton, J. J. Orteu, and H. Schreier, Image Correlation for Shape, Motion and Deformation. Springer, 2009.

[9] D. Lecompte et al., "Quality assessment of speckle patterns for digital image correlation," Opt. Lasers Eng., vol. 44, no. 11, pp. 1132-1145, Nov. 2006.

[10] Hild F. and Roux S., "Digital Image Correlation: from Displacement Measurement to Identification of Elastic Properties - a Review," Strain, vol. 42, no. 2, pp. 69-80, Apr. 2006.

[11] B. Pan, K. Qian, H. Xie, and A. Asundi, "Two-dimensional digital image correlation for inplane displacement and strain measurement: a review," Meas. Sci. Technol., vol. 20, no. 6, p. 062001, 2009.

[12] M. A. Sutton, N. Li, D. C. Joy, A. P. Reynolds, and X. Li, "Scanning Electron Microscopy for Quantitative Small and Large Deformation Measurements Part I: SEM Imaging at Magnifications from 200 to 10,000," Exp. Mech., vol. 47, no. 6, pp. 775-787, Dec. 2007.

[13] A. D. Kammers and S. Daly, "Self-Assembled Nanoparticle Surface Patterning for Improved Digital Image Correlation in a Scanning Electron Microscope," Exp. Mech., vol. 53, no. 8, pp. 1333-1341, Oct. 2013.

[14] B. Boen, "NASA Engineers Crush Giant Fuel Tank To Improve Rocket Designs," NASA, 15Apr-2015. [Online]. Available: http://www.nasa.gov/exploration/systems/sls/shellbuckling-test.html. [Accessed: 07-Sep-2017].

[15] J. T. Hammer, T. J. Liutkus, J. D. Seidt, and A. Gilat, "Using Digital Image Correlation (DIC) in Dynamic Punch Tests," Exp. Mech., vol. 55, no. 1, pp. 201-210, Jan. 2015.

[16] B. J. Murienne and T. D. Nguyen, "A comparison of 2D and 3D digital image correlation for a membrane under inflation," Opt. Lasers Eng., vol. 77, pp. 92-99, Feb. 2016. 
[17] P. F. Luo, Y. J. Chao, M. A. Sutton, and W. H. Peters, "Accurate measurement of threedimensional deformations in deformable and rigid bodies using computer vision," Exp. Mech., vol. 33, no. 2, pp. 123-132, Jun. 1993.

[18] E. López-Alba, L. Felipe-Sesé, S. Schmeer, and F. A. Díaz, “Optical low-cost and portable arrangement for full field 3D displacement measurement using a single camera," Meas. Sci. Technol., vol. 27, no. 11, p. 115901, 2016.

[19] J.-J. Orteu, "3-D computer vision in experimental mechanics," Opt. Lasers Eng., vol. 47, no. 3, pp. 282-291, Mar. 2009.

[20] M. Born and E. Wolf, Principles of Optics: Electromagnetic Theory of Propagation, Interference and Diffraction of Light. Elsevier, 2013.

[21] C. Neipp, A. Beléndez, S. Gallego, M. Ortuño, I. Pascual, and J. T. Sheridan, “Angular responses of the first and second diffracted orders in transmission diffraction grating recorded on photopolymer material," Opt. Express, vol. 11, no. 16, pp. 1835-1843, Aug. 2003.

[22] R. Magnusson and T. K. Gaylord, "Diffraction efficiencies of thin phase gratings with arbitrary grating shape," JOSA, vol. 68, no. 6, pp. 806-809, Jun. 1978.

[23] S. Larouche and D. R. Smith, "Reconciliation of generalized refraction with diffraction theory," Opt. Lett., vol. 37, no. 12, pp. 2391-2393, Jun. 2012.

[24] P. Lalanne, S. Astilean, P. Chavel, E. Cambril, and H. Launois, "Design and fabrication of blazed binary diffractive elements with sampling periods smaller than the structural cutoff," JOSA A, vol. 16, no. 5, pp. 1143-1156, May 1999.

[25] S. Xia, A. Gdoutou, and G. Ravichandran, "Diffraction Assisted Image Correlation: A Novel Method for Measuring Three-Dimensional Deformation using Two-Dimensional Digital Image Correlation," Exp. Mech., vol. 53, no. 5, pp. 755-765, Jun. 2013.

[26] M. Trivi and H. J. Rabal, "Stereoscopic uses of diffraction gratings," Appl. Opt., vol. 27, no. 6, p. 1007, Mar. 1988.

[27] B. Pan and Q. Wang, "Single-camera microscopic stereo digital image correlation using a diffraction grating," Opt. Express, vol. 21, no. 21, p. 25056, Oct. 2013.

[28] Z. Pan, S. Xia, A. Gdoutou, and G. Ravichandran, "Diffraction-Assisted Image Correlation for Three-Dimensional Surface Profiling," Exp. Mech., vol. 55, no. 1, pp. 155-165, Jan. 2015.

[29] B. M. B. Grant, H. J. Stone, P. J. Withers, and M. Preuss, "High-temperature strain field measurement using digital image correlation," J. Strain Anal. Eng. Des., vol. 44, no. 4, pp. 263-271, May 2009.

[30] M. D. Novak and F. W. Zok, "High-temperature materials testing with full-field strain measurement: Experimental design and practice," Rev. Sci. Instrum., vol. 82, no. 11, p. 115101, Nov. 2011.

[31] R. B. Berke and J. Lambros, "Ultraviolet digital image correlation (UV-DIC) for high temperature applications," Rev. Sci. Instrum., vol. 85, no. 4, p. 045121, Apr. 2014.

[32] P. Reu, "Virtual Strain Gage Size Study," Exp. Tech., vol. 39, no. 5, pp. 1-3, Sep. 2015.

[33] P. L. Reu, D. P. Rohe, and L. D. Jacobs, "Comparison of DIC and LDV for practical vibration and modal measurements," Mech. Syst. Signal Process., vol. 86, pp. 2-16, Mar. 2017. 
[34] G. G. Wells, N. Sampara, E. E. Kriezis, J. Fyson, and C. V. Brown, "Diffraction grating with suppressed zero order fabricated using dielectric forces," Opt. Lett., vol. 36, no. 22, pp. 4404-4406, Nov. 2011. 